\title{
Mesons on a transverse lattice
}

\author{
Simon Dalley \\ Centre for Mathematical Sciences, Cambridge University \\ Wilberforce Road, Cambridge CB3 OWA, England
}

\begin{abstract}
The meson eigenstates of the light-cone Hamiltonian in a coarse transverse lattice gauge theory are investigated. Building upon previous work in pure gauge theory, the Hamiltonian and its Fock space are expanded in powers of dynamical fields. In the leading approximation, the couplings appearing in the Hamiltonian are renormalised by demanding restoration of space-time symmetries broken by the cut-off. Additional requirements from chiral symmetry are discussed and difficulties in imposing them from first principles in the leading approximation are noted. A phenomenological calculation is then performed, in which chiral symmetry in spontaneously broken form is modelled by imposing the physical $\pi$ - $\rho$ mass splitting as a constraint. The light-cone wavefunctions of the resulting Hamiltonian are used to compute decay constants, form factors and quark momentum and spin distributions for the pion and rho mesons. Extensions beyond leading order, and the implications for first principles calculations, are briefly discussed.
\end{abstract}

Typeset using REVTEX 


\section{INTRODUCTION}

Many questions of a dynamical nature in QCD cannot easily be addressed in conventional lattice quantisation schemes [1,2]. Light-cone quantisation is a Hamiltonian framework suitable for highly-relativistic bound and scattering states [3, [4], and would be ideal for extending the kinds of problem that may be tackled. The corresponding light-cone wavefunctions are frame-invariant, contain no disconnected vacuum contributions, and matrix elements of suitable operators can be related to virtually any hadronic process. In this work, the light-cone Hamiltonian for a version of lattice QCD with quarks is investigated. It builds upon previous work of the author with B. van de Sande on the pure gauge theory [5 77, following an original idea of Bardeen et. al. [8,9]. The particular lattice gauge theory and its approximation considered here was first proposed in ref. [10].

All the transverse lattice treatments of non-abelian gauge theory cited above are formulated in terms of disordered link-fields $M$, which transform gauge covariantly but are not themselves restricted to the gauge group. As such, they are expected to be well-suited to lattice spacings $a$ that are coarse-enough (compared to $\Lambda_{\mathrm{QCD}}$ ) for the use of disordered elementary degrees of freedom. The question then arises as to how to determine the effective Hamiltonian. The expected level of coarseness probably rules out any reliable use of perturbation theory on the basis of asymptotic freedom. The Hamiltonian must be renormalised with a non-perturbative technique. Pure QCD may be defined by gauge and Poincaré symmetries, together with an explicit connection to the asymptotically free continuum limit. In transverse lattice gauge theory, one discretizes two (transverse) space directions on a lattice, say $\mathbf{x}=\left\{x^{1}, x^{2}\right\}$, while maintaining lattice gauge invariance. In refs. [6, [7] it was suggested that, since the other space-time directions $\left\{x^{0}, x^{3}\right\}$ remain continuous, tuning the Hamiltonian to restore Poincaré symmetry at finite transverse lattice spacing would be enough to study spectral quantities in QCD. An approximation scheme for implementing this idea is the colour-dielectric expansion. For sufficiently massive fields $M$, low-lying hadronic light-cone wavefunctions may converge rapidly in parton number, making expansion of the Hamiltonian in powers of $M$ viable. This method was tested and symmetry requirements alone produced a well-defined trajectory of couplings to leading order of the power expansion of the Hamiltonian. Glueball spectra on this trajectory scaled and agreed with results from other methods (the most accurate agreement has been found in $2+1$-dimensional studies [11]).

A similar method is pursued here in the light-meson sector, where a number of new features arise. In anticipation of a constituent structure appearing for boundstates when the ultra-violet cut-off is lowered sufficiently, expansion of the Hamiltonian in powers of dynamical fields, both disordered links and quarks, will again be the basis of the approximation scheme. In this work, the expansion is also extended to the Hilbert space itself; an additional Tamm-Dancoff cut-off will be put on the number of partons in a Fock space. The convergence of these approximations will not be studied here; rather, as a necessary first step, the lowest order of approximation will be solved in order to see if a reasonable phenomenology results at this level. The cut-off Hamiltonian will be renormalised non-perturbatively by searching the space of couplings for a region where spacetime symmetries violated by the cut-off are being restored. Unlike in pure gauge theory, however, one must also confront approximate chiral symmetry (i.e. one must fix the quark mass). Practical problems when trying to 
impose both Poincaré and chiral symmetry in the leading approximation are noted, which may require higher orders and/or renormalisation of operators other than the Hamiltonian to overcome. For this work, chiral symmetry in spontaneously broken form is therefore modelled by demanding the $\pi$ - $\rho$ mass splitting as a phenomenological constraint. With this caveat, a well-defined region in coupling space that enhances covariance of light-meson boundstates is found.

Using the bare expressions for currents, the light-cone eigenfunctions of the Hamiltonian renormalised and constrained in this way are used to compute decay constants, form factors and quark momentum and spin distributions of the pion and rho. These are compared with experimental results, where available, and other phenomenological and lattice results. The leading approximation is found to be a useful quantitative starting point for the calculation of such observables. Issues in going beyond the leading approximation and how this may allow one to use symmetry rigorously as a renormalisation method, are briefly discussed.

\section{TRANSVERSE LATTICE}

\section{A. Overview}

Light-cone quantisation is a Hamiltonian method that treats $x^{+}=\left(x^{0}+x^{3}\right) / \sqrt{2}$ as canonical time and $\left\{x^{-}=\left(x^{0}-x^{3}\right) / \sqrt{2}, \mathbf{x}\right\}$ as the spatial variables. Lorentz indices $\mu, \nu$ are split into light-cone indices $\alpha, \beta \in\{+,-\}$ and transverse indices $r, s \in\{1,2\}$. The metric has non-zero entries $g^{+-}=-g^{11}=-g^{22}=1$. In transverse lattice gauge theory, link variables $M_{r}\left(x^{+}, x^{-}, \mathbf{x}\right)$ are associated with the link from $\mathbf{x}$ to $\mathbf{x}+a \hat{\mathbf{r}}$ at fixed $x^{+}$and $x^{-}$. They physically represent the (averaged) flux between these points. Continuum $S U(N)$ gauge potentials $A_{\alpha}\left(x^{+}, x^{-}, \mathbf{x}\right)$ and fermions fields $\Psi\left(x^{+}, x^{-}, \mathbf{x}\right)$ are associated to a transverse plane $\mathbf{x}=$ const.. A slice through spacetime at fixed $x^{+}$is illustrated in figure 1 . The field variables transform under transverse lattice gauge transformations $V\left(x^{+}, x^{-}, \mathbf{x}\right) \in S U(N)$ associated with the transverse planes as

$$
\begin{aligned}
A_{\alpha}(\mathbf{x}) & \rightarrow V(\mathbf{x}) A_{\alpha}(\mathbf{x}) V^{\dagger}(\mathbf{x})+\mathrm{i}\left(\partial_{\alpha} V(\mathbf{x})\right) V^{\dagger}(\mathbf{x}) \\
M_{r}(\mathbf{x}) & \rightarrow V(\mathbf{x}) M_{r}(\mathbf{x}) V^{\dagger}(\mathbf{x}+a \hat{\mathbf{r}}) \\
\Psi(\mathbf{x}) & \rightarrow V(\mathbf{x}) \Psi(\mathbf{x})
\end{aligned}
$$

The most general transverse lattice Lagrangian $L$ will consist of all operators invariant under lattice gauge symmetries (2.1), Poincaré symmetries manifestly preserved by the lattice cutoff, and renormalisable by dimensional counting with respect to the continuum co-ordinates $x^{\alpha}$. It is important to note that $M$ need not be restricted to $S U(N)$ [8]. It can be taken as a general $N \mathrm{x} N$ complex matrix, provided it still gauge transforms covariantly.

For QCD, in the transverse continuum limit $a \rightarrow 0$ one expects the couplings in $L$ to run with $a$ such that $M$ is forced into $S U(N)$ [四]. Writing $M=H U$, where $H$ is an hermitian matrix, as the transverse continuum limit is approached, $H$ should be expanded about a vacuum value $H=H_{0}+\tilde{H}$. The fluctuation $\tilde{H}$ should be made infinitely heavy by $L$ as it runs to $a=0$. Unfortunately, the light-cone quantisation of non-linear degrees of freedom $U$ and vacuum modes $H_{0}$ is very awkard. Even if one formulates the theory entirely in terms of only unitary variables $U$ from the beginning, the light-cone quantisation is still highly 
non-trivial [12]. However, in the region of coupling space where $H_{0}=0, M$ behaves as a massive linear degree of freedom and light-cone quantisation is straightforward. The colourdielectric hypothesis suggests that the renormalised trajectory [13], on which sufficiently long-distance observables take the same values as in the continuum limit, passes into this region at sufficiently large $a$ [5, 14]. Since weak-coupling perturbation theory is unlikely to be reliable for the construction of this trajectory at large $a$, it was suggested to use symmetry to reconstruct it [7,:11]. In the case of a transverse lattice regulator, it is plausible that with the most general cut-off $L$, gauge and Poincaré symmetry are sufficient to identify a unique trajectory associated to QCD. Whether or not this is correct, it nevertheless motivates an approximate treatment of this idea.

In reality, one must truncate the number of operators appearing in the most general $L$ and also introduce further cut-offs on the Hilbert space, beyond the transverse lattice cut-off $a$. The subset of symmetry criteria one chooses are now obviously influenced by the approximations made. The truncation of $L$, in the presence of only the cut-off $a$, will be based upon the relative importance of different field configurations expected in low-lying hadronic boundstates. This substitutes for the fact that one cannot make use of dimensional counting with respect to transverse co-ordinates $\mathbf{x}$, since $a$ is not assumed to be small. It then makes sense to test symmetries only in the lowest-energy observables. Eventually, this procedure should be repeated, adding more operators to $L$ and testing symmetries at ever higher energies. It is possible that the approximation scheme employed, although systematic and physically motivated, is poor. That is, it restricts one to couplings to operators that poorly approximate a renormalised trajectory. This can only be decided by explicitly checking for restoration of symmetries violated by the cut-off. The approximations will be further modified by the presence of additional cut-offs on the Hilbert space. The simplest nontrivial approximation to the QCD Lagrangian and Hilbert space is used as the basis for an explicit computation in this paper. Issues in going beyond this approximation are also briefly discussed.

In the rest of this section, approximation of the Hamiltonian (II.B), Hilbert space (II.C), and chiral symmetry issues (II.D) are addressed. The calculation within the leading approximation is detailed in section III, first with the introduction of explicit symmetry criteria (III.A), then applications to phenomenology (III.B \& C).

\section{B. Approximating the Hamiltonian}

Starting from the most general transverse lattice Lagrangian $L$, we wish to arrive at a systematically improvable approximation to the light-cone Hamiltonian $P^{-}$. First, recall the (continuum) proper Poincaré generators in light-cone co-ordinates [15 for a set of singlecomponent fields labelled generically $\phi_{l}$. At a particular light-cone time $x^{+}$,

$$
\begin{aligned}
P^{\nu} & =\int d x^{-} d^{2} \mathbf{x} T^{+\nu} \\
M^{\mu \nu} & =\int d x^{-} d^{2} \mathbf{x}\left(x^{\mu} T^{+\nu}-x^{\nu} T^{+\mu}+\pi_{l} \Sigma_{l m}^{\mu \nu} \phi_{m}\right),
\end{aligned}
$$

where $T$ is the energy-momentum

$$
T^{+\mu}=\pi_{l} \partial^{\mu} \phi_{l}-g^{+\mu} L
$$


$\pi_{l}$ are the canonical momenta

$$
\pi_{l}=\frac{\delta L}{\delta\left(\partial_{+} \phi_{l}\right)},
$$

and the spin part for spinor or vector fields respectively is

$$
\Sigma_{l m}^{\mu \nu}=\frac{1}{4}\left[\gamma^{\mu}, \gamma^{\nu}\right]_{l m} \quad \text { or } \quad g_{l}^{\mu} g_{m}^{\nu}-g_{m}^{\mu} g_{l}^{\nu}
$$

The mass shell condition is

$$
2 P^{+} P^{-}=\mathcal{M}^{2}+\left(P^{1}\right)^{2}+\left(P^{2}\right)^{2} .
$$

There are also the discrete symmetries $\left\{P: x^{-} \leftrightarrow x^{+}, \mathbf{x} \rightarrow-\mathbf{x}\right\}$ and $\left\{T: x^{-} \leftrightarrow-x^{+}\right\}$, related to improper Lorentz transformations. For the moment, no chiral symmetry is assumed.

The (classical) space-time symmetries will be classified into violated or preserved, and kinematic or dynamic, in the presence of the transverse lattice cut-off $a$. The most general Lagrangian may only contain lattice gauge-invariant operators invariant under preserved Poincaré symmetries. In the continuum, the subset $\left\{P^{r}, P^{+}, M^{+r}, M^{12}, M^{-+}\right\}$can usually be chosen kinematic. That is, they can be chosen - at a particular $x^{+}$and in a particular gauge perhaps - to be independent of interactions, quadratic in independent dynamical fields. As many generators as possible will be maintained in kinematic form, consistent with lattice gauge invariance. The imposition of a lattice cut-off on transverse directions means that the continuous symmetries associated to $\left\{P^{r}, M^{12}, M^{+r}\right\}$ are violated (although there is a discrete subset of finite symmetry transformations that are preserved). Demanding that the generators $\left\{P^{+}, M^{-+}\right\}$of preserved symmetries can be chosen kinematic, they can easily be used to define states of non-zero longitudinal momentum. Although the symmetry generated by $M^{+r}$ is in general violated, at a particular time $x^{+}=0$ it is recovered and kinematic, and will be used to define transversely boosted states at this time. Of the remaining dynamic generators $\left\{P^{-}, M^{-r}\right\}$, the symmetry generated by the Hamiltonian $P^{-}$ is preserved, while that generated by the boost-rotations $M^{-r}$ is violated. Parity $P$ and time reversal $T$ are dynamic and preserved, though $P T$, hence charge congugation $C$ (or $G$-parity), is kinematic.

Demanding a kinematic $P^{+}$restricts the possible kinetic term for $L$ to

$$
\begin{aligned}
L_{\text {kin }}= & \sum_{\mathbf{x}} \int d x^{-} \sum_{\alpha, \beta=+,-} \sum_{r=1,2}-\frac{1}{2 G^{2}} \operatorname{Tr}\left\{F^{\alpha \beta} F_{\alpha \beta}\right\}+\mathrm{i} \bar{\Psi} \gamma^{\alpha}\left(\partial_{\alpha}+\mathrm{i} A_{\alpha}\right) \Psi \\
& +\operatorname{Tr}\left\{\left[\left(\partial_{\alpha}+\mathrm{i} A_{\alpha}(\mathbf{x})\right) M_{r}(\mathbf{x})-\mathrm{i} M_{r}(\mathbf{x}) A_{\alpha}(\mathbf{x}+a \hat{\mathbf{r}})\right][\text { h.c. }]\right\}
\end{aligned}
$$

in which h.c. is hermitian conjugate and light-cone gauge $A_{-}=0$ is chosen. This partial gauge fixing will render $A_{+}$non-dynamical, independent of $x^{+}$according to equations of motion; half the components of the fermion spinor, $\Psi^{-}=\gamma^{0} \gamma^{-} \Psi$, will also be non-dynamical (see below).

The potential term for $L$ will be expanded in powers of the dynamical fields $M, \Psi^{+}=$ $\gamma^{0} \gamma^{+} \Psi$ (after elimination of non-dynamical fields $A_{ \pm}$and $\Psi^{-}$). Such a power expansion can only be justified in a region of coupling space where these fields are sufficiently heavy 
that light-cone wavefunctions of interest converge quickly in parton number. For sufficiently heavy fields, the lowest-mass lattice hadrons will consist of a few partons $M$ and $\Psi_{+}$with little mixing into configurations with many partons. The physical motivation for expecting a renormalised trajectory to exist in the region of link-fields $M$ with positive mass squared is the the colour-dielectric picture of confinement [8,14]. The dynamical generation of a constituent quark mass in QCD makes this scenario physically plausible for quarks also. In both cases, the ultra-violet cut-off on the renormalised trajectory must be lowered sufficently for these constituent approximations to be viable. Dimensional counting in the continuous directions $\left(x^{+}, x^{-}\right)$, together with the power expansion in dynamical fields, limits the number of allowed operators to some extent. However, locality in $x^{-}$cannot be assumed. Elimination of non-dynamical degrees of freedom will inevitably introduce non-local terms. This could, in principle, lead to $x^{-}$-dependent couplings 4 . Demanding that $P^{-}$is derived from a gauge-invariant Lagrangian $L$ and that $P^{+}$be kinematic appears to eliminate this possibility however, at least at level of the lattice cut-off theory.

The simplest approximation to the Lagrangian in QCD is then

$$
\begin{aligned}
L= & \sum_{\mathbf{x}} \int d x^{-} \sum_{\alpha, \beta=+,-} \sum_{r=1,2}-\frac{1}{2 G^{2}} \operatorname{Tr}\left\{F^{\alpha \beta} F_{\alpha \beta}\right\} \\
& +\operatorname{Tr}\left\{\left[\left(\partial_{\alpha}+\mathrm{i} A_{\alpha}(\mathbf{x})\right) M_{r}(\mathbf{x})-\mathrm{i} M_{r}(\mathbf{x}) A_{\alpha}(\mathbf{x}+a \hat{\mathbf{r}})\right][\text { h.c. }]\right\} \\
& -\mu_{b}^{2} \operatorname{Tr}\left\{M_{r} M_{r}^{\dagger}\right\}+\mathrm{i} \bar{\Psi} \gamma^{\alpha}\left(\partial_{\alpha}+\mathrm{i} A_{\alpha}\right) \Psi-\mu_{f} \bar{\Psi} \Psi \\
& +\mathrm{i} \kappa_{a}\left(\bar{\Psi}(\mathbf{x}) \gamma^{r} M_{r}(\mathbf{x}) \Psi(\mathbf{x}+a \hat{\mathbf{r}})-\bar{\Psi}(\mathbf{x}) \gamma^{r} M_{r}^{\dagger}(\mathbf{x}-a \hat{\mathbf{r}}) \Psi(\mathbf{x}-a \hat{\mathbf{r}})\right) \\
& +\kappa_{s}\left(\bar{\Psi}(\mathbf{x}) M_{r}(\mathbf{x}) \Psi(\mathbf{x}+a \hat{\mathbf{r}})+\bar{\Psi}(\mathbf{x}) M_{r}^{\dagger}(\mathbf{x}-a \hat{\mathbf{r}}) \Psi(\mathbf{x}-a \hat{\mathbf{r}})\right)
\end{aligned}
$$

where $G, \mu_{f}, \mu_{b}, \kappa_{a}, \kappa_{s}$ are coupling constants and the $A_{-}=0$ gauge is taken. This will be the starting point for explicit calculations in this paper. Detailed investigation of the corrections to this $L$, involving higher powers of fields, is left to future work.

In the chiral representation (Appendix A-2 of ref. [16]), $\Psi^{\dagger}=\left(u_{+}^{*}, v_{+}^{*}, v_{-}^{*}, u_{-}^{*}\right) / 2^{1 / 4}$ decomposes into left (right) movers $v(u)$ with a helicity subscript $h= \pm$. In light-cone gauge $A_{-}=0, A_{+}$and $v_{ \pm}$satisfy constraint equations of motion which are used to eliminate them at the classical level. Defining

$$
\begin{aligned}
F_{h}(\mathbf{x})= & -u_{h}(\mathbf{x})+\frac{\kappa_{s}}{\mu_{f}} \sum_{r}\left(M_{r}(\mathbf{x}) u_{h}(\mathbf{x}+a \hat{\mathbf{r}})+M_{r}^{\dagger}(\mathbf{x}-a \hat{\mathbf{r}}) u_{h}(\mathbf{x}-a \hat{\mathbf{r}})\right) \\
& \frac{h \mathrm{i} \kappa_{a}}{\sqrt{2} \mu_{f}}\left\{\left(M_{1}(\mathbf{x})-h \mathrm{i} M_{2}(\mathbf{x})\right) u_{-h}(\mathbf{x}+a \hat{\mathbf{r}})\right. \\
& \left.-\left(M_{1}^{\dagger}(\mathbf{x}-a \hat{\mathbf{r}})+h \mathrm{i} M_{2}^{\dagger}(\mathbf{x}-a \hat{\mathbf{r}})\right) u_{-h}(\mathbf{x}-a \hat{\mathbf{r}})\right\} \\
J^{+}(\mathbf{x})= & \mathrm{i} \sum_{r}\left(M_{r}(\mathbf{x}) \overleftrightarrow{\partial}_{-} M_{r}^{\dagger}(\mathbf{x})+M_{r}^{\dagger}(\mathbf{x}-a \hat{\mathbf{r}}) \stackrel{\leftrightarrow}{\partial}_{-} M_{r}(\mathbf{x}-a \hat{\mathbf{r}})\right) \\
& +\sum_{h} u_{h}(\mathbf{x}) u_{h}^{\dagger}(\mathbf{x}),
\end{aligned}
$$

the constraints are

$$
\begin{aligned}
\left(\partial_{-}\right)^{2} A_{+} & =\frac{G^{2}}{2}\left(J^{+}-\frac{1}{N} \operatorname{Tr} J^{+}\right) \\
\mathrm{i} \partial_{-} v_{h} & =\frac{\mu_{f}}{\sqrt{2}} F_{-h} .
\end{aligned}
$$


Introducing gauge indices $\{i, j \in\{1,2, \cdots N\}\}$, the canonical momenta are $\pi_{i}\left(u_{h}\right)=\mathrm{i} u_{h, i}^{*}$, $\pi_{i j}\left(M_{r}\right)=\partial_{-} M_{r, i j}^{*}$. Hence, the generators of preserved symmetries at $x^{+}=0$ are

$$
\begin{aligned}
P^{-}= & \int d x^{-} \sum_{\mathbf{x}} \frac{G^{2}}{4}\left(\operatorname{Tr}\left\{J^{+} \frac{1}{\left(\mathrm{i} \partial_{-}\right)^{2}} J^{+}\right\}-\frac{1}{N} \operatorname{Tr}\left\{J^{+}\right\} \frac{1}{\left(\mathrm{i} \partial_{-}\right)^{2}} \operatorname{Tr}\left\{J^{+}\right\}\right) \\
& +\frac{\mu_{f}^{2}}{2} \sum_{h}\left(F_{h}^{\dagger} \frac{1}{\mathrm{i} \partial_{-}} F_{h}\right)+\mu_{b}^{2} \sum_{r=1}^{2} \operatorname{Tr}\left\{M_{r} M_{r}^{\dagger}\right\}, \\
P^{+}= & \int d x^{-} \sum_{\mathbf{x}, s, h} 2 \operatorname{Tr}\left\{\partial_{-} M_{s}(\mathbf{x}) \partial_{-} M_{s}(\mathbf{x})^{\dagger}\right\}+\mathrm{i} u_{h}^{*} \partial_{-} u_{h}, \\
M^{-+}= & \int d x^{-} \sum_{\mathbf{x}, s, h} x^{-}\left\{2 \operatorname{Tr}\left\{\partial_{-} M_{s}(\mathbf{x}) \partial_{-} M_{s}(\mathbf{x})^{\dagger}\right\}+\frac{\mathrm{i}}{2} u_{h}^{*} \stackrel{\leftrightarrow}{\partial}_{-} u_{h}\right\}, \\
M^{+r}= & -\int d x^{-} \sum_{\mathbf{x}, s, h} 2\left(x^{r}+\frac{a}{2} \delta^{r s}\right) \operatorname{Tr}\left\{\partial_{-} M_{s}(\mathbf{x}) \partial_{-} M_{s}(\mathbf{x})^{\dagger}\right\}+\mathrm{i} x^{r} u_{h}^{*} \partial_{-} u_{h} .
\end{aligned}
$$

The Hamiltonian (2.13) contains cubic and quartic interactions in dynamical fields. At quartic order there would be further terms, in addition to those in (2.13), generated from quartic terms allowed in the Lagrangian, that have been neglected in (2.8). The lowest order of the power expansion would retain only the cubic interactions in $P^{-}$, but this approximation would not produce confinement. One is free to include some or all of the possible terms at quartic order in $P^{-}$. As a minimal prescription, only the quartic terms arising from the $J^{+} J^{+}$interaction will be retained, as they are be responsible for confinement [8]. The effect of other quartic terms is left for future investigation; in particular, it is likely that they will be important for parity and chiral symmetry restoration, as discussed later.

\section{Cut-offs in Fock space}

In the quantum theory, there are (anti-)commutation relations at fixed $x^{+}$

$$
\begin{aligned}
{\left[M_{r, i j}\left(x^{-}, \mathbf{x}\right), \partial_{-} M_{s, k l}^{*}\left(y^{-}, \mathbf{y}\right)\right] } & =\frac{\mathrm{i}}{2} \delta_{i k} \delta_{j l} \delta\left(x^{-}-y^{-}\right) \delta_{\mathbf{x y}} \delta_{r s}, \\
\left\{u_{h, i}\left(x^{-}, \mathbf{x}\right), \mathrm{i} u_{h^{\prime}, j}^{*}\left(y^{-}, \mathbf{y}\right)\right\} & =\mathrm{i} \delta_{i j} \delta\left(x^{-}-y^{-}\right) \delta_{\mathbf{x y}} \delta_{h h^{\prime}} .
\end{aligned}
$$

A convenient Fock space representation employs longitudinal momentum space but transverse position space

$$
\begin{aligned}
M_{r}\left(x^{+}=0, x^{-}, \mathbf{x}\right) & =\frac{1}{\sqrt{4 \pi}} \int_{0}^{\infty} \frac{d k^{+}}{\sqrt{k^{+}}}\left(a_{-r}\left(k^{+}, \mathbf{x}\right) e^{-\mathrm{i} k^{+} x^{-}}+a_{r}^{\dagger}\left(k^{+}, \mathbf{x}\right) e^{\mathrm{i} k^{+} x^{-}}\right), \\
{\left[a_{\lambda, i j}\left(k^{+}, \mathbf{x}\right), a_{\rho, k l}^{*}\left(\tilde{k}^{+}, \mathbf{y}\right]\right.} & =\delta_{i k} \delta_{j l} \delta_{\lambda \rho} \delta_{\mathbf{x y}} \delta\left(k^{+}-\tilde{k}^{+}\right), \\
{\left[a_{\lambda, i j}\left(k^{+}, \mathbf{x}\right), a_{\rho, k l}\left(\tilde{k}^{+}, \mathbf{y}\right)\right] } & =0 . \\
u_{h}\left(x^{+}=0, x^{-}, \mathbf{x}\right) & =\frac{1}{\sqrt{2 \pi}} \int_{0}^{\infty} \frac{d k^{+}}{\sqrt{k}}\left(b_{h}\left(k^{+}, \mathbf{x}\right) e^{-\mathrm{i} k^{+} x^{-}}+d_{-h}^{*}\left(k^{+}, \mathbf{x}\right) e^{\mathrm{i} k^{+} x^{-}}\right), \\
\left\{b_{h, i}\left(k^{+}, \mathbf{x}\right), b_{h^{\prime}, j}^{*}\left(\tilde{k}^{+}, \mathbf{y}\right\}\right. & =\delta_{i j} \delta_{h h^{\prime}} \delta_{\mathbf{x y}} \delta\left(k^{+}-\tilde{k}^{+}\right), \\
\left\{b_{h, i}\left(k^{+}, \mathbf{x}\right), b_{h^{\prime}, j}\left(\tilde{k}^{+}, \mathbf{y}\right)\right\} & =0 .
\end{aligned}
$$


Here, $\lambda$ and $\rho \in\{ \pm 1, \pm 2\}, a_{\lambda, i j}^{*}=a_{\lambda, j i}^{\dagger}$, and similar anti-commutators exist for $d$. The Fock space operator $a_{r, i j}^{\dagger}\left(k^{+}, \mathbf{x}\right)$ creates a link-parton with longitudinal momentum $k^{+}$, carrying colour $i$ at $\mathbf{x}$ to $j$ at $\mathbf{x}+a \widehat{\mathbf{r}} . b_{h, i}^{*}\left(k^{+}, \mathbf{x}\right)$ creates a quark of helicity $h$, colour $i$, momentum $k^{+}$at site $\mathbf{x}$, while $d^{*}$ does the same for anti-quarks. This Fock space is already diagonal in $P^{+}$and serves as a basis for finding the eigenvalues of $P^{-}$.

The $1 / \partial_{-}$non-local terms in $P^{-}$(2.13) cause divergences at small $k^{+}$. However, the well-known elimination of such divergences by a suitable normal-ordering prescription in $1+1$-dimensional gauge and Yukawa theories [17] carries over to the transverse lattice Hamiltonian at finite $a$. While the currents $J^{+}$and $F$ are normal-ordered, the Hamiltonian $P^{-}$itself is not. This generates infinite self-inertias for the $M$ and $\Psi^{+}$partons, which are required for finiteness of the eigenvalues of $P^{-}$. Moreover, as can be seen from (2.13), the finite-energy physical states $|\psi\rangle$ satisfy

$$
\int d x^{-} J^{+}|\psi\rangle=\int d x^{-} F_{ \pm}|\psi\rangle=\int d x^{-} F_{ \pm}^{\dagger}|\psi\rangle=0
$$

The $J^{+}$condition singles out Fock states invariant under residual $x^{-}$-independent gauge transformations $V(\mathbf{x})$ in the light-cone gauge [8]. For these singlet configurations, there is linear confinement of heavy sources for generic coupling choices in the region of sufficiently large $\mu_{b}^{2}$ 18. The $F$ conditions will relate Fock space wavefunction components at small $k^{+}$ momentum with different numbers of partons, and are the source of rising (Regge) behaviour of structure functions at small Bjorken $x$ [19.

In order to render the Fock space finite-dimensional, suitable for study on a computer, it is necessary to impose further cut-offs. DLCQ [17,20 will be used to discretize longitudinal momentum, by compactifying $x^{-}$on circle of circumference $\mathcal{L}=2 \pi K / P^{+}$, where $K$ is a positive integer, with (anti)-periodic boundary conditions for $M(u)$. All $k^{+}=0$ modes will be dropped, whether or not the boundary conditions allow it. The DLCQ cut-off is applied directly to the Poincaré generators and results extrapolated to $K=\infty$. However, since the zero modes are not picked up explicitly by the $K \rightarrow \infty$ extrapolation, further finite renormalisation due to their absence is expected. General arguments about the kinds of finite renormalisation expected upon omission of zero modes in light-cone QCD have been given in ref. [4]. Previously preserved dynamical symmetries could in general be violated by DLCQ without zero modes, even in the limit $K \rightarrow \infty$. In particular, parity invariance is violated by zero mode omission and some work has been done on the corresponding renormalisation required in the context of simpler field theories [21]. This is one place where quark 'kinetic' mass terms can persist, even in a chiral theory, leading to a viable constituent approximation. The quartic terms neglected in $P^{-}$(2.13) are needed to address parity restoration in general.

There are also zero modes associated to the transverse and longitudinal gauge fields. To justify the power expansion, the link-field mass $\mu_{b}^{2}$ was taken sufficently large. This ensures that the $k^{+}=0$ mode of the (radial part of the) transverse fields $M$ is pushed to infinite energy, so one may indeed expand about $M=0$ (2.19). On the other hand, the omission of zero modes of $A^{\alpha}$ could lead to additional finite renormalisations for zero momentum transfer 4 -parton interactions induced by the instantaneous gluon exchange kernel $1 / \partial_{-}^{2}$. While the

\footnotetext{
${ }^{1}$ This justifies post hoc the fact that boundary terms were not discussed earlier.
} 
zero mode of $A_{+}$can be gauged away at a particular $x^{+}$, the zero mode of $A_{-}$cannot be gauge fixed to zero in the presence of periodic $x^{-}$boundary conditions [22]. There has been some study of the effects of explicitly retaining such a mode in simpler field theories [23], which indicate that there may be a small effect in the instantaneous gluon exchange between $M$ and $\Psi^{+}$. Parity symmetry again would be the obvious place to look for fixing these zeromode induced effects. No attempt to incorporate further zero-mode induced operators into $P^{-}$will be made here.

Although DLCQ at fixed $K$ and without zero modes automatically cuts off the maximum number of partons in Fock space, it will be convenient to impose a separate Tamm-Dancoff cut-off on this number. Ideally, this cut-off would be extrapolated, along with the DLCQ one, as has been done in transverse lattice studies of pure gauge theory [7]. In the present work, the Tamm-Dancoff cut-off will be fixed at the smallest non-trivial value throughout the calculation for simplicity. In the 'one-link' truncation of Fock space, a general $\mathbf{P}=\mathbf{0}$ normalised state can be expressed in terms of orthonormal Fock states as

$$
\begin{aligned}
\left|\psi\left(P^{+}\right)\right\rangle= & \frac{1}{\sqrt{\mathrm{Vol}}} \sum_{\mathbf{x}} a^{2} \sum_{h, h^{\prime}} \int_{0}^{1} d x_{1} d x_{2} \delta\left(x_{1}+x_{2}-1\right) \\
& \times\left\{\psi_{h h^{\prime}}\left(x_{1}, x_{2}\right) N^{-1 / 2} d_{h}^{\dagger}\left(x_{1}, \mathbf{x}\right) b_{h^{\prime}}^{*}\left(x_{2}, \mathbf{x}\right)|0\rangle\right\} \\
& +\frac{1}{\sqrt{\mathrm{Vol}}} \sum_{\mathbf{x}} a^{2} \sum_{h, h^{\prime}, r} \int_{0}^{1} d x_{1} d x_{2} d x_{3} \delta\left(x_{1}+x_{2}+x_{3}-1\right) \\
& \times\left\{\psi_{h(r) h^{\prime}}\left(x_{1}, x_{2}, x_{3}\right) N^{-1} d_{h}^{\dagger}\left(x_{1}, \mathbf{x}\right) a_{r}^{\dagger}\left(x_{2}, \mathbf{x}\right) b_{h^{\prime}}^{*}\left(x_{3}, \mathbf{x}+a \hat{\mathbf{r}}\right)|0\rangle\right. \\
& \left.+\psi_{h(-r) h^{\prime}}\left(x_{1}, x_{2}, x_{3}\right) N^{-1} d_{h}^{\dagger}\left(x_{1}, \mathbf{x}+a \hat{\mathbf{r}}\right) a_{-r}^{\dagger}\left(x_{2}, \mathbf{x}\right) b_{h^{\prime}}^{*}\left(x_{3}, \mathbf{x}\right)|0\rangle\right\}
\end{aligned}
$$

where Vol is the volume of transverse space; see figure 1 . The $M_{-+}$boost-invariant momentum fractions $x_{1}=k_{1}^{+} / P^{+}$etc. have been introduced, which should not be confused with transverse co-ordinates. DLCQ amounts to discretizing such fractions into integer or odd half-integer units of $1 / K$. The wavefunction $\left|\psi\left(P^{+}\right)\right\rangle$automatically satisfies the $k^{+}$ momentum and helicity sum rules, even at finite $K$.

States of non-zero $\mathbf{P}$ are straightforwardly generated by application of $M_{-r}$

$$
\begin{aligned}
\psi_{h h^{\prime}}\left(x_{1}, x_{2}\right) & \rightarrow \exp [\mathrm{i} \mathbf{P} . \mathbf{x}] \psi_{h h^{\prime}}\left(x_{1}, x_{2}\right) \\
\psi_{h(r) h^{\prime}}\left(x_{1}, x_{2}, x_{3}\right) & \rightarrow \exp \left[\mathrm{i} \mathbf{P} .\left(x_{1} \mathbf{x}+x_{2}(\mathbf{x}+0.5 a \hat{\mathbf{r}})+x_{3}(\mathbf{x}+a \hat{\mathbf{r}})\right)\right] \psi_{h(r) h^{\prime}}\left(x_{1}, x_{2}, x_{3}\right) \\
\psi_{h(-r) h^{\prime}}\left(x_{1}, x_{2}, x_{3}\right) & \rightarrow \exp \left[\mathrm{i} \mathbf{P} .\left(x_{1}(\mathbf{x}+a \hat{\mathbf{r}})+x_{2}(\mathbf{x}+0.5 a \hat{\mathbf{r}})+x_{3}(\mathbf{x})\right)\right] \psi_{h(-r) h^{\prime}}\left(x_{1}, x_{2}, x_{3}\right) .
\end{aligned}
$$

A fixed Tamm-Dancoff approximation will obviously lead to Fock-sector-dependent renormalisations; in the present case this means that $\mu_{f}$ is sector-dependent. The analysis of later sections was performed both with and without the assumption of sector dependence. The main conclusions are qualitatively similar, but only results with a sector-independent mass $\mu_{f}$ are shown, since it was possible to sweep the coupling space with greater resolution in this case.

In the Fock space (2.27), the $x^{-}$global colour-singlet states have already been picked out by applying the $J^{+}$constraint (2.25), since it simplifies the DLCQ Fock space. (Application of the $F$ condition does not simplify the DLCQ Fock space particularly, so is not imposed from the beginning; the constraint will be satisfied dynamically when $P^{-}$is diagonalised). 
In the one-link truncation, a quark and antiquark either share the same transverse lattice site, or are separated by one link. It is the simplest approximation that still allows a meson to propagate on the transverse lattice. A correct description of the favour-singlet sector would require inclusion of quark - anti-quark annihilation processes, which are forbidden in the one-link approximation. Therefore, the space (2.27) will be interpreted as appropriate to the flavour-non-singlet mesons, by identifying the quark and anti-quark with (degenerate) up and down flavours respectively. No flavour label is necessary in this case, and flavour is assumed to have been summed over in operators.

Projecting $2 P^{+} P^{-}|\psi(\mathbf{P}=\mathbf{0})\rangle$ onto Fock basis states, one derives the following set of coupled integral equations for individual Fock components:

$$
\begin{aligned}
& \frac{\mathcal{M}^{2}}{\bar{G}^{2}} \psi_{h h^{\prime}}\left(x_{1}, x_{2}\right)=\left(\frac{m_{f}^{2}}{x_{1}}+\frac{m_{f}^{2}}{x_{2}}\right) \psi_{h h^{\prime}}\left(x_{1}, x_{2}\right)+K\left(\psi_{h h^{\prime}}\left(x_{1}, x_{2}\right)\right) \\
& +\frac{2\left(k_{a}^{2}+k_{s}^{2}\right)}{\pi}\left(\frac{1}{x_{1}} \int_{0}^{x_{1}} \frac{d y}{y}+\frac{1}{x_{2}} \int_{0}^{x_{2}} \frac{d y}{y}\right) \psi_{h h^{\prime}}\left(x_{1}, x_{2}\right) \\
& -\sum_{\lambda}\left\{\frac{m_{f} k_{s}}{\sqrt{2 \pi}} \int_{0}^{x_{1}} \frac{d y}{\sqrt{y}}\left(\frac{1}{x_{1}-y}+\frac{1}{x_{1}}\right) \psi_{h(\lambda) h^{\prime}}\left(x_{1}-y, y, x_{2}\right)\right. \\
& +\frac{m_{f} k_{s}}{\sqrt{2 \pi}} \int_{0}^{x_{2}} \frac{d y}{\sqrt{y}}\left(\frac{1}{x_{2}-y}+\frac{1}{x_{2}}\right) \psi_{h(\lambda) h^{\prime}}\left(x_{1}, y, x_{2}-y\right) \\
& +\frac{m_{f} k_{a}\left(h \mathrm{i} \operatorname{Sgn}(\lambda) \delta_{|\lambda| 1}+\delta_{|\lambda| 2}\right)}{\sqrt{2 \pi}} \int_{0}^{x_{1}} \frac{d y}{\sqrt{y}}\left(\frac{1}{x_{1}-y}-\frac{1}{x_{1}}\right) \psi_{-h(\lambda) h^{\prime}}\left(x_{1}-y, y, x_{2}\right) \\
& \left.+\frac{m_{f} k_{a}\left(h^{\prime} \mathrm{i} \operatorname{Sgn}(\lambda) \delta_{|\lambda| 1}-\delta_{|\lambda| 2}\right)}{\sqrt{2 \pi}} \int_{0}^{x_{2}} \frac{d y}{\sqrt{y}}\left(\frac{1}{x_{2}-y}-\frac{1}{x_{2}}\right) \psi_{h(\lambda)-h^{\prime}}\left(x_{1}, y, x_{2}-y\right)\right\}, \\
& \frac{\mathcal{M}^{2}}{\bar{G}^{2}} \psi_{h(\lambda) h^{\prime}}\left(x_{1}, x_{2}, x_{3}\right)=\left(\frac{m_{b}^{2}}{x_{2}}+\frac{m_{f}^{2}}{x_{1}}+\frac{m_{f}^{2}}{x_{3}}\right) \psi_{h(\lambda) h^{\prime}}\left(x_{1}, x_{2}, x_{3}\right)+K\left(\psi_{h(\lambda) h^{\prime}}\left(x_{1}, x_{2}, x_{3}\right)\right) \\
& -\frac{m_{f} k_{s}}{\sqrt{2 \pi x_{2}}}\left(\frac{1}{x_{1}}+\frac{1}{x_{1}+x_{2}}\right) \psi_{h h^{\prime}}\left(x_{1}+x_{2}, x_{3}\right) \\
& -\frac{m_{f} k_{s}}{\sqrt{2 \pi x_{2}}}\left(\frac{1}{x_{3}}+\frac{1}{x_{2}+x_{3}}\right) \psi_{h h^{\prime}}\left(x_{1}, x_{2}+x_{3}\right) \\
& -\operatorname{Sgn}(\lambda) \frac{\left(h \mathrm{i} \delta_{|\lambda| 1}+\delta_{|\lambda| 2}\right) m_{f} k_{a}}{\sqrt{2 \pi x_{2}}}\left(\frac{1}{x_{1}}-\frac{1}{x_{1}+x_{2}}\right) \psi_{-h h^{\prime}}\left(x_{1}+x_{2}, x_{3}\right) \\
& -\operatorname{Sgn}(\lambda) \frac{\left(h^{\prime} \mathrm{i} \delta_{|\lambda| 1}-\delta_{|\lambda| 2}\right) m_{f} k_{a}}{\sqrt{2 \pi x_{2}}}\left(\frac{1}{x_{3}}-\frac{1}{x_{2}+x_{3}}\right) \psi_{h-h^{\prime}}\left(x_{1}, x_{2}+x_{3}\right) .
\end{aligned}
$$

The conventions of ref. 24 have been adopted for the instantaneous gluon kernels

$$
\begin{gathered}
K\left(\psi_{h h^{\prime}}\left(x_{1}, x_{2}\right)\right)=\frac{1}{2 \pi} \int_{0}^{1} d y\left\{\frac{\psi_{h h^{\prime}}\left(x_{1}, x_{2}\right)-\psi_{h h^{\prime}}(y, 1-y)}{\left(y-x_{1}\right)^{2}}\right\}, \\
K\left(\psi_{h(\lambda) h^{\prime}}\left(x_{1}, x_{2}, x_{3}\right)\right)=\frac{1}{2 \pi} \int_{0}^{x_{2}+x_{3}} d y \frac{\left(x_{3}+2 x_{2}-y\right)}{2\left(x_{3}-y\right)^{2} \sqrt{x_{2}\left(x_{2}+x_{3}-y\right)}}\left\{\psi_{h(\lambda) h^{\prime}}\left(x_{1}, x_{2}, x_{3}\right)\right.
\end{gathered}
$$




$$
\begin{aligned}
& \left.-\psi_{h(\lambda) h^{\prime}}\left(x_{1}, x_{2}+x_{3}-y, y\right)\right\} \\
& +\frac{1}{2 \pi x_{3}}\left(\sqrt{1+\frac{x_{3}}{x_{2}}}-1\right) \psi_{h(\lambda) h^{\prime}}\left(x_{1}, x_{2}, x_{3}\right) \\
& +\frac{1}{2 \pi} \int_{0}^{x_{1}+x_{2}} d y \frac{\left(x_{1}+2 x_{2}-y\right)}{2\left(x_{1}-y\right)^{2} \sqrt{x_{2}\left(x_{1}+x_{2}-y\right)}}\left\{\psi_{h(\lambda) h^{\prime}}\left(x_{1}, x_{2}, x_{3}\right)\right. \\
& \left.-\psi_{h(\lambda) h^{\prime}}\left(y, x_{1}+x_{2}-y, x_{3}\right)\right\} \\
& +\frac{1}{2 \pi x_{1}}\left(\sqrt{1+\frac{x_{1}}{x_{2}}}-1\right) \psi_{h(\lambda) h^{\prime}}\left(x_{1}, x_{2}, x_{3}\right) .
\end{aligned}
$$

$\bar{G}=G \sqrt{\left(N^{2}-1\right) / N}$, which has the dimensions of mass, and the following renormalisations have been introduced:

$$
\frac{\mu_{b}}{\bar{G}} \rightarrow m_{b} \quad ; \quad \frac{\mu_{f}}{\bar{G}} \rightarrow m_{f} \quad ; \frac{\kappa_{a} \sqrt{N}}{\bar{G}} \rightarrow k_{a} \quad ; \quad \frac{\kappa_{s} \sqrt{N}}{\bar{G}} \rightarrow k_{s} .
$$

The dimensionless variables $\left\{m_{b}, m_{f}, m_{f} k_{s}, m_{f} k_{a}\right\}$ appearing in the Hamiltonian equations (2.29) (2.30) are to be understood as the renormalised couplings after imposing DLCQ and Tamm-Dancoff cut-offs on the Fock space. All $N$-dependence of the theory has now been absorbed into the couplings.

\section{Chiral symmetry}

Chiral symmetries are subtle both on a lattice and in light-cone quantisation. Consider first the effects of the lattice cut-off. The lattice Lagrangian (2.8) explicitly breaks the chiral symmetry

$$
\Psi \rightarrow e^{-\mathrm{i} \theta \gamma_{5}} \Psi
$$

through the bare mass-term $\mu_{f}$ and Wilson term $\kappa_{s}$. The latter is needed to eliminate fermion doublers in the free-fermion dispersion.

When chiral symmetry is not explicitly broken at the Lagrangian level (a chiral theory), spontaneous breaking would be signalled by vacuum degrees of freedom in a conventional quantisation scheme. The light-cone problem with small $k^{+}$regulated does not have such explicit degrees of freedom in the light-cone vacuum. There are two, somewhat related, mechanisms one could envisage for the manifestation of spontaneously broken chiral symmetry in this case. Zero modes, being vacuum degrees of freedom, may carry information about the spontaneous breaking of chiral symmetry, and their omission may require one to add counter-terms to $P^{-}$that explicitly break chiral symmetry, even if it is only broken spontaneously in the conventional sense [1]. Spontaneous breaking may occur in a more-orless conventional way through wee partons that enhance contributions from operators that break chiral symmetry in the limit that their couplings are sent to zero [25]. An explicit illustration of the latter effect was given in ref. [26]. Note that, once a Tamm-Dancoff cut-off is introduced, the effects of both these mechanisms on the Hamiltonian would be similar. The wee partons are excluded by this cut-off, so the net result of the second mechanism would be to introduce finite couplings to chiral-symmetry breaking counterterms in the cut-off theory. 
At the Lagrangian level, the chiral limit is $\mu_{f} \rightarrow 0$ and $\kappa_{s} \rightarrow 0$. However, the renormalised parameters $\left\{m_{f}^{2}, m_{f} k_{s}, m_{f} k_{a}\right\}$ need not be zero in the chiral limit. To address chiral symmetry at this level, one can introduce the light-cone chiral transformation [4]

$$
\Psi^{+} \rightarrow e^{-\mathrm{i} \theta \gamma_{5}} \Psi^{+}
$$

where $\Psi=\left(\Psi^{+}+\Psi^{-}\right) / \sqrt{2}$ has been defined earlier, and $\Psi^{-}$is determined through the constraint equation (2.12). This is equivalent to the usual chiral transformation on $\Psi$ (2.34) only in a chiral theory. Omitting zero modes, the light-cone chiral charge that generates (2.35) measures helicity

$$
\begin{aligned}
Q_{5} & =\int d x^{-} \sum_{\mathbf{x}} \bar{\Psi} \gamma^{+} \gamma_{5} \Psi \\
& =\int d x^{-} \sum_{\mathbf{x}} \sum_{h} h u_{h}^{*} u_{h} .
\end{aligned}
$$

It is equivalent to the usual chiral charge only in a chiral theory without spontaneous breaking, the difference presumably lying in contributions at $x^{-}$infinity. In a chiral theory with spontaneous breaking of the symmetry and no explicit small $k^{+}$degrees of freedom to reflect this, one therefore expects helicity-violating operators in the renormalised Hamiltonian. In the Hamiltonian equations (2.29) 2.30), only the operator coupling to $m_{f} k_{a}$ breaks the helicity symmetry (2.35).

Conservation of the renormalised axial current $A_{\mu}$ defines a chiral theory. One may use PCAC to impose chiral symmetry on the theory, through the matrix element

$$
\left\langle 0\left|\partial_{\mu} A_{\mu}\right| \psi_{\pi}\left(P^{\mu}\right)\right\rangle=f_{\pi} \mathcal{M}_{\pi}^{2}
$$

Without knowing the precise expression for $A_{\mu}$ in terms of bare fields, chiral symmetry can be imposed by setting either $f_{\pi}=0$ or $\mathcal{M}_{\pi}=0$. To test for a phase of gauge theory with spontaneously broken chiral symmetry, one would then search the space of couplings for a region where Poincaré symmetry is restored, $\mathcal{M}_{\pi}=0$, and there is no instability. In the leading approximation considered in this paper, $m_{f} k_{a}$ couples to the only hopping term that propagates the $\pi$ and $\rho$ at different rates on the transverse lattice. In the absence of any other hopping term that distinguishes the pion and rho, Poincaré symmetries would require this coupling to be as small as possible, i.e. as small as stability of the theory allows. $m_{f} k_{a}$ is also the only source of mass difference between the pion and rho. Unfortunately, stability is difficult to check from first principles in the one-link approximation. Any instability in the quantum field theory would almost certainly be signalled by copious parton production, which is forbidden in the one-link approximation.

Because of the difficulty in checking for instability in the approximation made in this paper, a phenomenological constraint will be introduced that is intended to model the effects of spontaneously broken chiral symmetry in the one link approximation. First, the experimental string tension $\sigma$ will be used to set the QCD scale. This quantity was calculated in terms of lattice quantities in ref. [7]. Then both the $\pi$ and $\rho$ masses will be fit

\footnotetext{
${ }^{2}$ These calculations involved no modelling, using Poincaré symmetry and the power expansion alone. The large- $N$ limit was also taken. However, the present calculation is independent of $N$ in the one-link approximation up to a trivial redefinition of $G . G \sqrt{N}$ in ref. [7] is identified with $\bar{G}$.
} 
to the experimental values. This involves three fits to experimental numbers, when QCD itself has only two parameters (e.g. the quark mass and $\Lambda_{Q C D}$ ). Thus there is a single phenomenological constraint. The use of $\pi$ and $\rho$ masses is intended to model the fact that (a) a nearly chiral theory in the Goldstone mode should have a light pion and (b) helicity violation, that splits the $\pi$ and $\rho$ masses, should be present in $P^{-}$when chiral symmetry is broken only spontaneously. The remaining freedom in the couplings present in $P^{-}$will be fixed by optimizing meson dispersion. This, together with the use of a more accurate calculation of $\sigma$, represents the main advance over the purely phenomenological modelling of this lattice gauge theory performed in ref. [10].

\section{MODEL CALCULATIONS}

\section{A. Symmetry Tests}

The one-link approximation is too simple to allow much change in a meson's size in lattice units, while retaining its physical size and covariant dispersion. Therefore, the spacing $a$ will be fixed at the largest value that gave reasonable results in ref. [7], by setting $m_{b}=0.2$. In this case $\bar{G} \approx 3.11 \sqrt{\sigma}$ and $a \bar{G} \approx 4.57$. If one takes $\sqrt{\sigma}=440 \mathrm{MeV}$, then $\bar{G}=1368 \mathrm{MeV}$ and $a \approx(300 \mathrm{MeV})^{-1} \equiv 2 / 3 \mathrm{fm}$. This is obviously the maximum quark separation in the one-link approximation.

Since the Hamiltonian (2.13) is transversely local, it makes sense to expand eigenvalues thus

$$
2 P^{+} P^{-}=\bar{G}^{2}\left(\mathcal{M}_{0}^{2}+\mathcal{M}_{1}^{2} a^{2} \mathbf{P}^{2}+O\left(a^{4} \mathbf{P}^{4}\right)\right)
$$

A necessary condition for Poincaré invariance is isotropy of the dispersion, i.e. the speed of light $c$ in transverse directions should satisfy

$$
a^{2} \bar{G}^{2} \mathcal{M}_{1}^{2} \equiv c^{2}=1
$$

It makes no sense to try to tune the anharmonic terms in (3.1) separately, since only nearest neighbour hopping is available in the current approximation.

The transverse lattice has symmetry group $D_{4}$ [9], with one-dimensional representations and a single two-dimensional irreducible representation. Since there is $90^{\circ}$ rotational symmetry about $x^{3}$, it is possible to distinguish the angular momentum projections $J_{3} \bmod 4$. The one-dimensional representations corresponds to $J_{3}=0$ or symmetric and antisymmetric combinations of $J_{3}= \pm 2$. The two-dimensional contains $J_{3}= \pm 1$. Generically, the pion is split from the rho, while the $J_{3}=0$ component of the rho $\left(\rho_{0}\right)$ is split from its degenerate

$J_{3}= \pm 1$ components $\left(\rho_{ \pm}\right)$. However, all these states become degenerate in the absence of quark helcity violating interactions. The zero-link sector serves to distinguish the various meson components, since they do not mix when $\mathbf{P}=\mathbf{0}$ as a result of $D_{4}$ symmetry.

$$
\begin{aligned}
& \pi: \psi_{+-}=-\psi_{-+} ; \psi_{ \pm \pm}=0 . \\
& \rho_{0}: \psi_{+-}=\psi_{-+} ; \psi_{ \pm \pm}=0 . \\
& \rho_{ \pm}: \psi_{ \pm \pm} \neq 0 ; \psi_{+-}=\psi_{-+}=0 .
\end{aligned}
$$


The three-dimensional space of dimensionless couplings $\left\{m_{f}, k_{a}, k_{s}\right\}$ was sampled discretely and the eigenvalue problem for $2 P^{+} P^{-}$solved for various $\mathbf{P}$. A $\chi^{2}$-test was introduced for the conditions (3.2) on the $\pi$ and $\rho$ dispersion and for the conditions that their masses assume the physical values, $\mathcal{M}_{\pi}=140 \mathrm{MeV}$ and $\mathcal{M}_{\rho}=770 \mathrm{MeV}$;

$$
\chi^{2}=w_{1}\left|\frac{\mathcal{M}_{\pi}}{\mathcal{M}_{\rho}}-0.18\right|+w_{2}\left|\frac{\mathcal{M}_{\rho}}{\bar{G}}-0.56\right|+\sum_{\pi, \rho_{ \pm}, \rho_{0}}|c-1| .
$$

Masses are defined as $\bar{G} \mathcal{M}_{0}$ from (3.1), where $\mathcal{M}_{\rho}$ is taken to be the average of the $\rho_{0}$ and $\rho_{ \pm}$masses. The weights $w_{1}$ and $w_{2}$ were adjusted to allow tolerance of about $100 \mathrm{MeV}$ in satisfying the mass conditions. The following results used $w_{1}=w_{2}=10$. Calculations were performed for DLCQ cut-offs $K=6,7,8,9$, with the corresponding bases of dimension $264,364,480,612$. The size of Fock space is quite modest and there is ample room for larger and more efficient calculations to be performed.

Figure 2 shows the results of the $\chi^{2}$ test. A clear minimum appears for every coupling at every $K$. While the details will change slightly with the specific test used, the broad features remain the same. To indicate what can be achieved at the minima of the $\chi^{2}$ shown in fig. 2 , at $K=9$ for example, the observables are shown in Table 1. For later calculations, it is convenient to fit the mid-point of the $\chi^{2}$ valley at each $K$ to a smooth form $A+B / K$,

$$
\begin{aligned}
m_{f} & =0.362(108)-\frac{0.969}{K}, \\
k_{a} & =0.162(927)+\frac{8.34}{K}, \\
k_{s} & =-0.323(921)+\frac{8.29}{K} .
\end{aligned}
$$

This will represent the best estimate of the symmetry-restoring trajectory at each $K$, for the range $K=6 \rightarrow 9$. When fitting to polynomials in $1 / K$, the magnitude of the last term in the series will be taken as an estimate of the error on the $K \rightarrow \infty$ result (the zeroth order term of the polynomial). Obviously, the fit to the couplings (3.6) does not produce a very useful estimate of the their $K \rightarrow \infty$ limit. However, the forms (3.6) are only used in the range $K=6 \rightarrow 9$ to recompute observables at those values, with a view to extrapolation of the observables themselves, whose convergence is typically faster than that of the couplings.

The observables were also fit to linear or quadratic polynomials in $1 / K$. A series in $1 / K$ is the correct form for the errors introduced by DLCQ discretization of the integral equations $(2.29)(2.30)$, if the integrands are analytic and the couplings are polynomial in $1 / K$. The integrands are in general not analytic, which will also produce non-integral powers of $1 / K$ in the finite- $K$ errors. However, the values of $K$ used here are too few and too small to warrant this level of complication in fitting functions. P

The light-cone eigenfunctions were recomputed along the smooth trajectory (3.6) and used to derive a number of observables for the $\pi$ and $\rho$. The observables are usually quoted

\footnotetext{
${ }^{3}$ The errors introduced by extrapolation in $K$ may make DLCQ seem inefficient compared to a basis of smooth wavefunctions. However, DLCQ is superficially easier to extend to multi-parton Fock states, which must be the eventual goal.
} 
at some transverse scale, which in the case of the tranvserse lattice is provided by $Q=$ $\pi / a \sim 1 \mathrm{GeV}$, the normalisation scale at which the non-perturbative wavefunctions have been determined. Observables will in general evolve with this scale, but $a$ has been fixed for the present calculation. The relationship between this scale and those introduced in other quantisation schemes ( $\overline{\mathrm{MS}}$ etc.) is non-trivial and will not be addressed here. For estimates, $Q$ will be identified with the momentum transfer from the probe where appropriate.

\section{B. Distribution amplitudes}

Distribution amplitudes for the $\pi$ and $\rho$ are defined by

$$
\begin{aligned}
\left.\left\langle 0\left|\bar{\Psi}(z) \gamma^{\mu} \gamma_{5} \Psi(0)\right| \psi_{\pi}\left(P^{\mu}\right)\right\rangle\right|_{z^{2}=0} & =f_{\pi} P^{\mu} \int_{0}^{1} \mathrm{e}^{\mathrm{i} x(z \cdot P)} \phi_{\pi}(x), \\
\left.\left\langle 0\left|\bar{\Psi}(z) \gamma^{\mu} \Psi(0)\right| \psi_{\rho_{0}}\left(P^{\mu}\right)\right\rangle\right|_{z^{2}=0} & =f_{\rho_{0}} P^{\mu} \int_{0}^{1} \mathrm{e}^{\mathrm{i} x(z \cdot P)} \phi_{\rho_{0}}(x), \\
\left.\left\langle 0\left|\bar{\Psi}(z) \sigma_{\mu \nu} \Psi(0)\right| \psi_{\rho_{ \pm}}\left(P^{\mu}\right)\right\rangle\right|_{z^{2}=0} & =f_{\rho_{ \pm}}\left(\epsilon_{\mu}^{ \pm}(P) P_{\nu}-\epsilon_{\nu}^{ \pm}(P) P_{\mu}\right) \int_{0}^{1} \mathrm{e}^{\mathrm{i} x(z . P)} \phi_{\rho_{ \pm}}(x),
\end{aligned}
$$

where the $\phi$ 's are normalised to one and $\epsilon$ is the polarization vector. They govern the leadingorder perturbative QCD expression for many high momentum transfer exclusive processes [27]. (The operators appearing in matrix elements above, and in other expressions later, will in general require further renormalisation due to the presence of cut-offs. For the purposes of the estimates made in this paper, the bare operators will be used.) For the pion, one finds

$$
\psi_{+-}(x, 1-x)=\frac{\pi f_{\pi}}{\sqrt{N}} \phi_{\pi}(x)
$$

from the $\gamma^{+}$component of (3.7); this is the component least likely to involve large renormalisations since it is quadratic in dynamical $\Psi^{+}$fields and involves only the continuum momentum $P^{+}$. Figure 3 shows this distribution amplitude at various $K$. A cubic interpolating function is used to produce a curve from the discrete values of momentum fraction $x$ sampled in DLCQ at each $K$. The resulting curves are extrapolated pointwise with a fit to a quadratic in $1 / K$, to yield the data points with extrapolation error bars shown also in figure 3. These extrapolated data points have been fit to the first two terms of the conformal expansion [28,29]

$$
\phi_{\pi}(x)=6 x(1-x)\left\{1+0.133 C_{2}^{3 / 2}\left(1-2 x^{2}\right)\right\} .
$$

The overall normalisation yields $f_{\pi}=98(13) \mathrm{MeV}$, compared to the experimental value $f_{\pi}$ (exp.) $=131 \mathrm{MeV}$. Because of error in the $\mathrm{MeV}$ scale setting, in the extrapolation in $K$, in the proportion of valence quarks in the pion, and/or in renormalisation, the result is about 30 percent too low in the current approximation, compared to the real world.

Direct experimental measurements of $\phi_{\pi}(x)$ have recently become possible from diffractive dissociation on a nucleus $\pi+A \rightarrow A+$ jets [30]. These experiments are carried out at somewhat larger $Q^{2} \sim 10 \mathrm{GeV}^{2}$, to minimize initial state interactions. Although the normalisation, which is $Q^{2}$-independent, is not correct, leading-order perturbative QCD evolution with boundary condition (3.11) produces a curve with shape consistent with the 
data measured in ref. [30]. The moments of $\phi_{\pi}$ have also been estimated from QCD sum rules. The most recent results [31] indicate a distribution amplitude close to the asymptotic form $x(1-x)$, even at low scales; this result is completely different from the early sum rule calculations of Chernyak and Zhitnistky [32], who suggested a large positive $C_{2}^{3 / 2}$ coefficient. The second moment of $\phi_{\pi}$ has also been calculated from first principles in Euclidean lattice QCD [33]. Although there is some systematic variation in those results, assuming the form

(3.11), the most recent lattice results suggest a somewhat negative $C_{2}^{3 / 2}$ coefficient! This also appears consistent with the E791 data, provided the coefficient is not too large.

An analysis for the $\rho$ yields very similar shapes and normalisations for the distributions. This means that there is a large deviation from experiment in the decay constant. For the helicity zero component of the rho, for example, one finds $f_{\rho_{0}}=89(14) \mathrm{MeV}$, less than half the experimental value $f_{\rho_{0}}$ (exp.) $=216 \mathrm{MeV}$. The rho meson distribution amplitude seems to be missing some essential physics at this level of approximation.

\section{Parton distributions}

The higher Fock state structure of the light-cone wavefunctions in general plays a role in any low momentum transfer process as well as high momentum transfer inclusive processes. For example, deep inelastic scattering probes the parton distribution function, the probability for finding a parton carrying certain longitudinal moment fraction of the hadron:

$$
V\left(x, Q^{2}\right)=\sum_{h, h^{\prime}}\left|\psi_{h h^{\prime}}(1-x, x)\right|^{2}+\sum_{\lambda} \sum_{h, h^{\prime}} \int_{0}^{1-x} d y\left|\psi_{h(\lambda) h^{\prime}}(1-x-y, y, x)\right|^{2} .
$$

The quark distribution function calculated in the pion is shown in figure 4 . In this case a linear extrapolation in $1 / K$ was necessary to achieve a smooth distribution. Also shown is a relevant phenomenological distribution that neglects sea quarks [34,

$$
V(x, 0.23)=\frac{0.526}{x^{0.495}}(1+0.357 \sqrt{x})(1-x)^{0.365},
$$

which was produced by fitting experimental $\pi N$ scattering data, after perturbative evolution to higher scales. (Note there is a slight difference in $Q^{2}$ scale). Although the calculated parton distribution appears close to the one of ref. [34], the excess at large $x$ means that the calculated total quark momentum fraction (the first moment of $V(x)), 0.43(1)$, is higher than typical experimental estimates [35]. Similarly, it is higher than estimates from quenched Euclidean lattice calculations of the low moments of $V$ [36], which yield about 0.3 for the first moment (at a slightly higher scale). This is consistent with the fact that the neglect of higher numbers of links in the one-link approximation excludes many gluon channels, so the quarks must pick up the momentum instead.

The pion elastic electromagnetic form factor depends upon a coherent overlap of the entire light-cone wavefunction at low momentum transfer:

$$
\left\langle\psi\left(P^{\prime}\right)\left|\int d x^{-} e^{\mathrm{i} q^{+} x^{-}} \sum_{\mathbf{x}} e^{\mathrm{iq} \cdot \mathbf{x}} \sum_{\eta} e_{\eta} \bar{\Psi}_{\eta} \gamma^{\mu} \Psi_{\eta}\right| \psi(P)\right\rangle=F\left(-q^{2}\right) \frac{\left(P^{\prime}+P\right)^{\mu}}{2},
$$


where $q=P^{\prime}-P$ is the exchanged photon's four-momentum and a flavour label $\eta$ and charges $e_{\eta}$ have been explicitly introduced to avoid confusion. The space-like form factor $F\left(-q^{2}\right),-q^{2}>0$, can be computed most simply from the + component of the current in the Drell-Yan frame $q^{+}=0$ [38], in which the photon's momentum is purely transverse. One finds

$$
\begin{aligned}
F\left(-q^{2}\right)= & \int_{0}^{1} d x_{1} d x_{2} \sum_{h, h^{\prime}} \delta\left(x_{1}+x_{2}-1\right)\left|\psi_{h h^{\prime}}\left(x_{1}, x_{2}\right)\right|^{2} \\
& +2 \sum_{h, h^{\prime}, r} \int_{0}^{1} d x_{1} d x_{2} d x_{3} \delta\left(x_{1}+x_{2}+x_{3}-1\right) \operatorname{Cos}\left(\left(x_{1}+\frac{x_{2}}{2}\right) a \mathbf{q} \cdot \hat{\mathbf{r}}\right)\left|\psi_{h(r) h^{\prime}}\left(x_{1}, x_{2}, x_{3}\right)\right|^{2} .
\end{aligned}
$$

$G$-parity and transverse reflection symmetries have been used to simplify the result. The resulting form factor, with q along a lattice axis, is shown in figure 5 (the calculated $F$ suffers from lattice artifacts and so is not rotationally invariant). The charge radius, given by $r_{\pi}^{2}=$ $6\left(\partial F\left(-q^{2}\right) / \partial q^{2}\right)_{q^{2}=0}$, will obviously be artificially reduced compared to the experimental value, $r_{\pi}=0.663 \mathrm{fm}$ [39]. This distance is roughly the same as one lattice spacing, which is the maximum quark separation in the one-link approximation. This explains why the curve in fig.5 is flatter than the true one [40]; the slope at the origin gives a charge radius about half the experimental value.

The quark distribution functions in the $\rho$ are shown in figure 6. One interesting feature, which may persist in more accurate calculations, is a difference between the longitudinal and transversely polarized $\rho$. This is consistent with some of the $\rho$ 's spin coming from orbital angular momentum of the quarks. In fact, one can directly measure the quark spin distribution in a transversely polarized rho meson $\rho_{ \pm}$(it is identically zero in a $\rho_{0}$ ). Define the helicity asymmetry

$$
A(x)=\frac{V_{+}(x)-V_{-}(x)}{V_{+}(x)+V_{-}(x)},
$$

where $V_{ \pm}(x)$ is the probability for a quark with helicty aligned or anti-aligned with that of the polarized $\rho$ respectively; $V(x)=V_{+}(x)+V_{-}(x)$. Figure 7 shows that a large part of the $\rho$ spin is not carried by the quark spins; one finds $s=0.41(10)$ for the quark spin fraction,

$$
s=\int_{0}^{1} d x\left(V_{+}(x)-V_{-}(x)\right)
$$

In the non-relativistic quark model, $s=1$. About half the rho's spin therefore must come from orbital angular momentum and gluon spin. Furthermore, fig. 7 exhibits the phenomena of helicity retention - the quark tends to align its spin with that of the hadron at large $x$ - which is expected on general grounds [37]. At small $x$ the quark helicity tends to become disordered, and the asymmetry $A(x)$ may even change sign eventually [19]. Euclidean lattice estimates of the low moments of the rho parton distributions [36] are consistent with the above results, except for the fact that, as with the pion, too much momentum is carried by quarks in the one-link approximation.

There are a number of subtleties in extracting the electromagnetic form factors of vector mesons from the light-cone wavefunctions; see for example, the discussion in ref. [41]. Since realistic values will in any case not be obtained in the one-link approximation, an analysis will not be attempted here for the $\rho$. 


\section{CONCLUSIONS}

Knowledge of the light-cone wavefunctions provides evident advantages over other methods, which determine low moments of operator matrix elements. Apart from renormalisation of the operators themselves, which is common to all methods, no further significant effort is required to calculate the matrix elements once the light-cone wavefunctions are determined. Moreover, these matrix elements are determined directly as functions of the momenta involved. On the other hand, identical low moments can give rise to functions of very different shape and moment integrals can often be very sensitive to inaccessible corners of phase space.

This paper has begun a program to calculate the light-cone wavefunctions of conventional hadrons from a transverse lattice gauge theory, using symmetry principles to renormalise the light-cone Hamiltonian. Expanding the most general Hamiltonian and the Fock space in powers of dynamical fields, the leading approximation led to practical difficulties in imposing simultaneously both Poincaré and chiral symmetry from first principles. While Poincaré symmetry could be tested through dispersion and PCAC could be imposed through $\mathcal{M}_{\pi}$, tests for stability of the theory would be compromised by the one-link approximation. In the $\pi-\rho$ sector, the mass splitting was therefore used as a phenomenological constraint to model the realisation of chiral symmetry in spontaneously broken form. The condition of isotropic dispersion then proved sufficient to unambiguously fix the remaining couplings appearing in the leading approximation. The resulting calculation in a Fock space restricted to at most one link reproduced a number of features of the pion and rho, known from experiment or estimated from Euclidean lattice calculations.

The next steps, which must go beyond the simplest approximations made here, will allow two questions to be addressed. Can symmetry principles alone be used to unambiguously renormalise the transverse lattice theory within a given approximation? Does the approximation scheme converge rapidly-enough to be of practical use? By extending the Tamm-Dancoff cut-off beyond one-link, it becomes easier to detect instabilities in the theory. As discussed in section II.D, this is one means by which chiral symmetry could be addressed from first principles — requiring a massless pion in a stable, Poincaré invariant theory. Beyond the one-link approximation it also becomes meaningful to add further quartic operators to the Hamiltonian. This will introduce further sources of parity and helicity violation that may be used to restore some of the parity and chiral symmetry broken by cut-offs. The Poincaré and chiral symmetry restoration should then be demonstrated over a range of lattice spacings $a$, as has been done for Poincaré symmetries in the case of pure gauge theories.

From a phenomenological point of view, the one-link approximation, with a lattice spacing of $2 / 3 \mathrm{fm}$, artificially squashes light mesons. By adding more links, allowing these mesons to expand to their physical size, observables such as form factors and quark momentum fractions should move closer to their experimental values. It will also become reasonable to include $P$-wave mesons in the analysis, with more symmetry constraints coming from their dispersion. Moreover, the simplest approximation for propagating baryons on the transvere lattice will require configurations with two links. 
Acknowledgements: I thank M. Burkardt for useful discussions. This work was supported by PPARC grant GR/LO3965. 


\section{REFERENCES}

[1] K. G. Wilson, Phys. Rev. D 10, 2445 (1974).

[2] J. B. Kogut and L. Susskind, Phys. Rev. D 11, 395 (1975).

[3] S. J. Brodsky, H.-C. Pauli, and S. Pinsky, Phys. Rep. 301, 299 (1998).

[4] K. G. Wilson et. al., Phys. Rev. D 49, 6720 (1994).

[5] S. Dalley and B. van de Sande, Phys. Rev. D 56, 7917 (1997).

[6] S. Dalley and B. van de Sande, Phys. Rev. D 59, 065008 (1999).

[7] S. Dalley and B. van de Sande, Phys. Rev. Lett. 82, 1088 (1999); Phys. Rev. D 62, 014507 (2000).

[8] W. A. Bardeen and R. B. Pearson, Phys. Rev. D 14, 547 (1976).

[9] W. A. Bardeen, R. B. Pearson, and E. Rabinovici, Phys. Rev. D 21, 1037 (1980).

[10] M. Burkardt and H. El-Khozondar, Phys. Rev. D 60, 054504 (1999).

[11] S. Dalley and B. van de Sande, hep-lat/0010082, to appear in Phys. Rev. D.

[12] P. A. Griffin, Nucl. Phys. B 139, 270 (1992).

[13] K. G. Wilson, in Recent Developments of Gauge Theories, eds. G. 't Hooft et al., Plenum New York (1980).

[14] G. Mack, Nucl. Phys. B 235, 197 (1984);

H. B. Nielsen and A. Patkos, Nucl. Phys. B 195, 137 (1982);

H.-J. Pirner, J. Wroldsen, and M. Ilgenfritz, Nucl. Phys. B 294, 905 (1987);

B. Grossman et. al., Int. J. Mod. Phys. A 6, 2649 (1991);

H.-J. Pirner, Prog. Part. Nucl. Phys. 29, 33 (1992).

[15] J. B. Kogut and D. E. Soper, Phys. Rev. D 1, 2901 (1970).

[16] C. Itzykson and J-B. Zuber, Quantum Field Theory, McGraw-Hill (1987).

[17] H.-C. Pauli and S. J. Brodsky, Phys. Rev. D 32, 1993 (1985); ibid 2001.

[18] M. Burkardt and B. Klindworth, Phys. Rev. D 55, 1001 (1997).

[19] F. Antonuccio, S. J. Brodsky, and S. Dalley, Phys.Lett. B 412, 104 (1997).

[20] A. Casher, Phys. Rev. D 14, 452 (1976);

T. Maskawa and K. Yamawaki, Prog. Theor. Phys. 56, 270 (1976).

C. Thorn, Phys. Lett. B 70, 77 (1977).

[21] M. Burkardt, Phys. Rev. D 54, 2913 (1996).

[22] V. A. Franke, Yu. A. Novozhilov, and E. V. Prokhvatilov, Lett. Math. Phys. 5, 239 (1981); ibid 437;

F. Lenz em et. al., Ann. Phys. 208, 1 (1991);

F. Lenz, H. W. L. Naus, M. Thies, Ann. Phys. 233, 317 (1994);

H-C. Pauli, A. C. Kalloniatis, and S. Pinsky, Phys. Rev. D 52, 1176 (1995).

[23] A. S. Mueller, A. C. Kalloniatis, and H-C. Pauli, Phys. Lett. B 435, 189 (1998).

[24] F. Antonuccio and S. Dalley, Phys. Lett. B 376, 154 (1996).

[25] A. Casher and L. Susskind, Phys. Rev. D 9, 436 (1974).

[26] M. Burkardt, Phys. Rev. D 58, 096015 (1998);

M. Burkardt and H. El-Khozondar, Phys. Rev. D 55, 6514 (1997).

[27] S. J. Brodsky and G. P. Lepage, in Perturbative Quantum Chromodynamics, ed. A. H. Mueller, (World Scientific Singapore 1989).

[28] G. P. Lepage and S. J. Brodsky, Phys. Lett. B 87, 359 (1979); Phys. Rev. D 22, 2157 (1980). 
[29] A. V. Efremov and A. V. Radyushkin, Phys. Lett. B 94, 245 (1980); Theor. Math. Phys. 42, 97 (1980).

[30] E791 Collaboration, E. M. Aitala et. al., hep-ex/0010043.

[31] S. V. Mikhailov and A. V. Radyushkin, Phys.Rev. D 45, 1754 (1992);

A. P. Bakulev and S. V. Mikhailov, Phys.Lett. B 436, 351 (1998);

V. M. Braun, A. Khodjamirian, and M. Maul, Phys.Rev. D 61, 073004 (2000).

[32] V. L. Chernyak and A. R. Zhitnitksy, Nucl. Phys. B 201, 492 (1982); B 214, 547(E) (1983); Phys. Rep. 112, 173 (1984)

[33] G. Martinelli and C. Sachrajda, Phys. Lett. B 190, 51 (1987); Nucl. Phys. B 306, 865 (1988);

T. DeGrand and R. D. Loft, Phys. Rev. D 38, 954 (1988);

T. Daniel, R. Gupta, and D. Richards, Phys. Rev. D 43, 3715 (1991);

L. Del Debbio et. al., Nucl. Phys. B (Proc. Suppl.) 83, 235 (2000).

[34] M. Glück, E. Reya, and A. Vogt, Z. Phys. C 53, 651 (1992).

[35] NA3 Collaboration, J. Badier et al., Z. Phys. C 28, 15 (1985).

[36] G. Martinelli and C. T. Sachrajda, Phys. Lett. B 196, 184 (1987);

C. Best et. al., Phys. Rev. D 56, 2743 (1997)

[37] S. J. Brodsky, M. Burkardt, and I. Schmidt, Nucl. Phys. B 441, 197 (1995).

[38] S. D. Drell and T. M. Yan, Phys. Rev. Lett. 24, 181 (1970).

[39] S. R. Amendolia et. al., Nucl. Phys. B 277, 168 (1986).

[40] C. J. Bebek et. al., Phys. Rev. D 13, 25 (1976).

[41] J. P. B. C. de Melo and T. Frederico, Phys. Rev. C 55, 2043 (1997). 


\section{TABLES}

\begin{tabular}{|c|cccc|}
\hline$K=9$ & $\pi$ & $\rho_{0}$ & $\rho_{+}$ & $\rho_{-}$ \\
\hline $\mathcal{M}(\mathrm{MeV})$ & 153 & 848 & 601 & 601 \\
$c$ & 1.18 & 0.73 & 0.69 & 1.16 \\
\hline
\end{tabular}

TABLE I. Observables at the minimum $\chi^{2}$ for $K=9$. 


\section{CAPTIONS}

FIGURE 1 - Transverse lattice (solid lines) at fixed $x^{+}$and meson configurations in a one-link approximation. Chain lines represent $P \exp \int d x^{-} A_{-}$required for gauge invariance.

FIGURE $2-\chi^{2}$ as a function of couplings for $m_{b}=0.2$ and $K=6,7,8,9$.

FIGURE 3 - The distribution amplitude $\psi(x)=\psi_{+-}=-\psi_{-+}$of the pion, at a transverse normalisation scale $Q^{2} \sim 1 \mathrm{GeV}^{2}$. Grey curves correspond to DLCQ cut-offs $K=6,7,8,9$ (darker means larger $K$ ). Data points are the pointwise extrapolation of finite- $K$ curves. The solid curve fits these data to the conformal expansion (3.11).

FIGURE 4 - The quark distribution function in the pion at a transverse normalisation scale $Q^{2} \sim 1 \mathrm{GeV}^{2}$. Grey curves correspond to DLCQ cut-offs $K=6,7,8,9$ (darker means larger $K$ ). Data points are the pointwise extrapolation of finite- $K$ curves. The solid curve (GRV) comes from a phenomenological fit to $\pi N$ scattering data [34], at normalisation scale $0.23 \mathrm{GeV}^{2}$.

FIGURE 5 - The pion electromagnetic form factor $F\left(-q^{2}\right)$. Grey curves correspond to DLCQ cut-offs $K=6,7,8,9$ (darker means larger $K$ ). Data points are the pointwise extrapolation of finite- $K$ curves.

FIGURE 6 - The quark distribution function in the longitudinally polarized $(h=0)$ and transversely polarized $(h=1)$ rho meson, linearly extrapolated in $1 / K$.

FIGURE 7 - The quark helicity asymmetry $A(x)$ in a transversely polarized $\rho$, linearly extrapolated in $1 / K$. 
FIGURES 
Figure 1

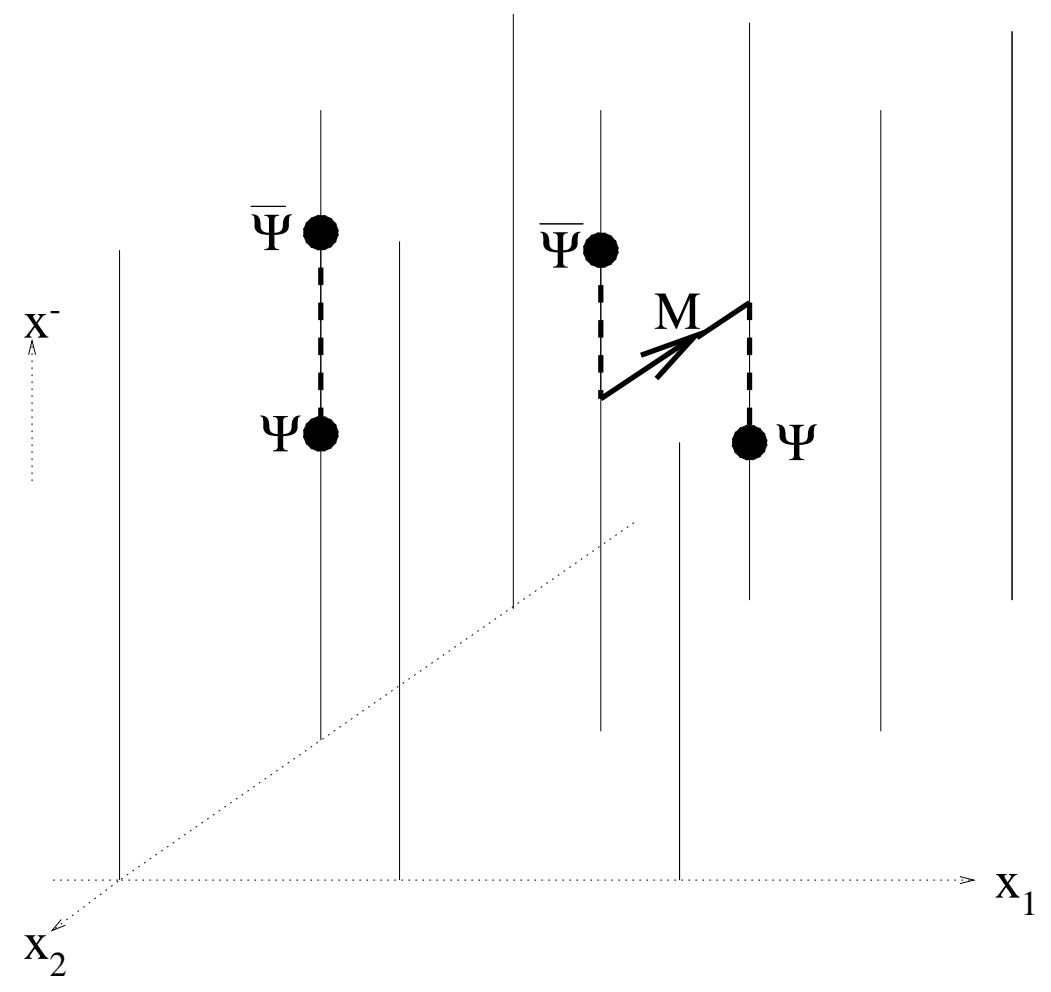


Figure 2
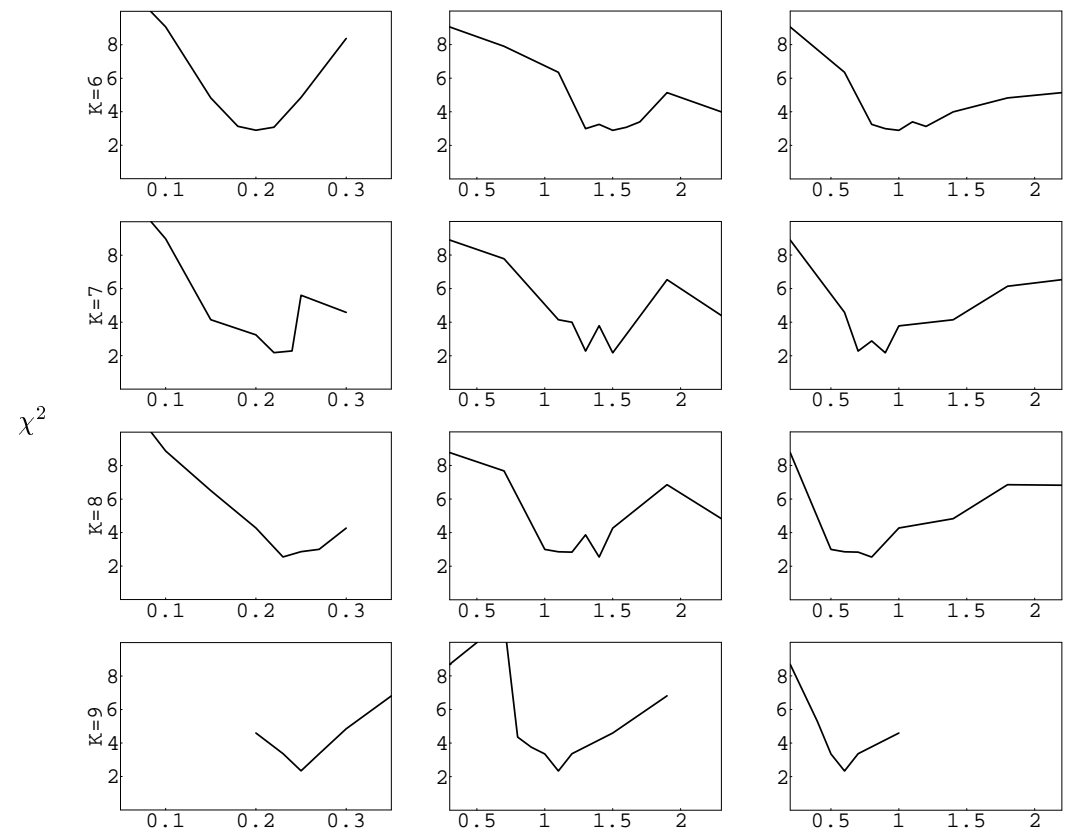

$m_{f}$

$k_{a}$

$k_{s}$ 
Figure 3

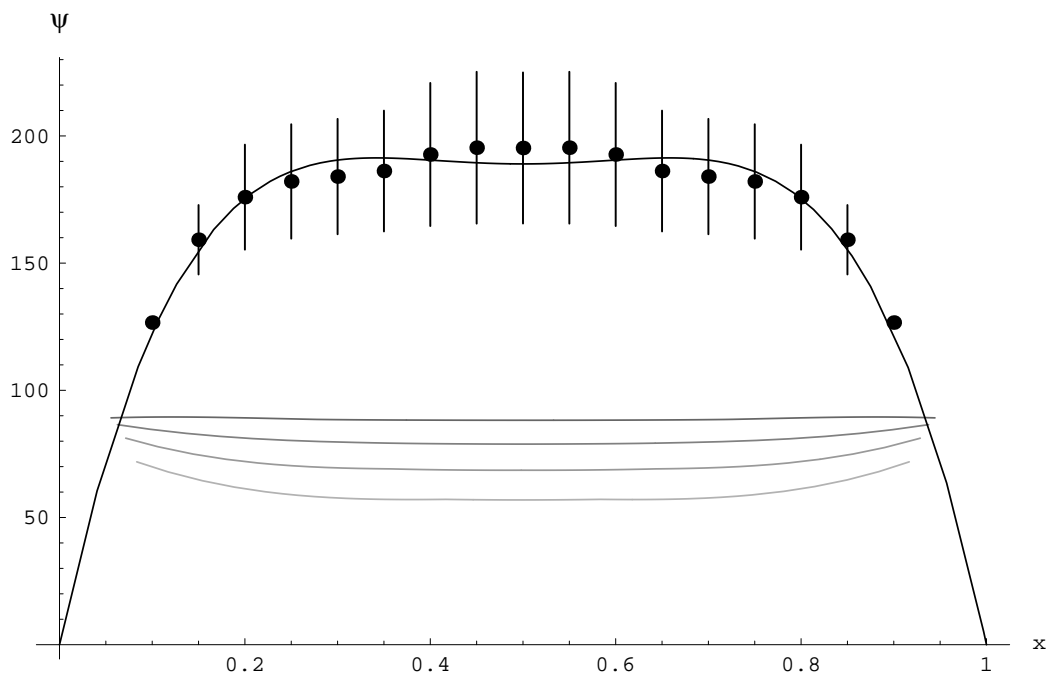


Figure 4

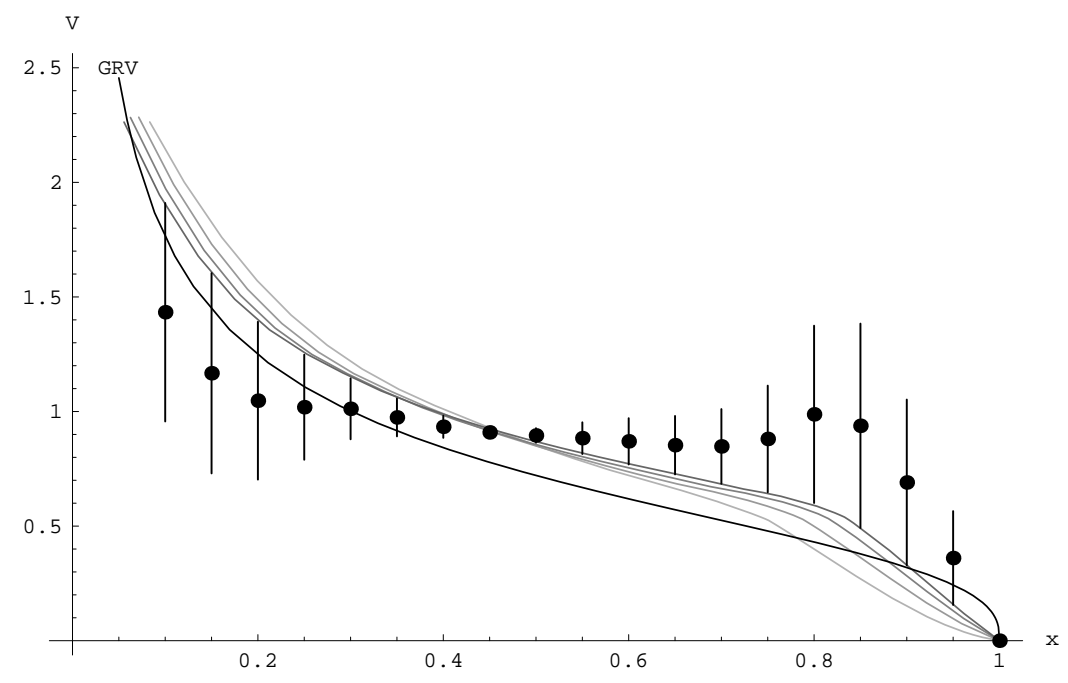


Figure 5

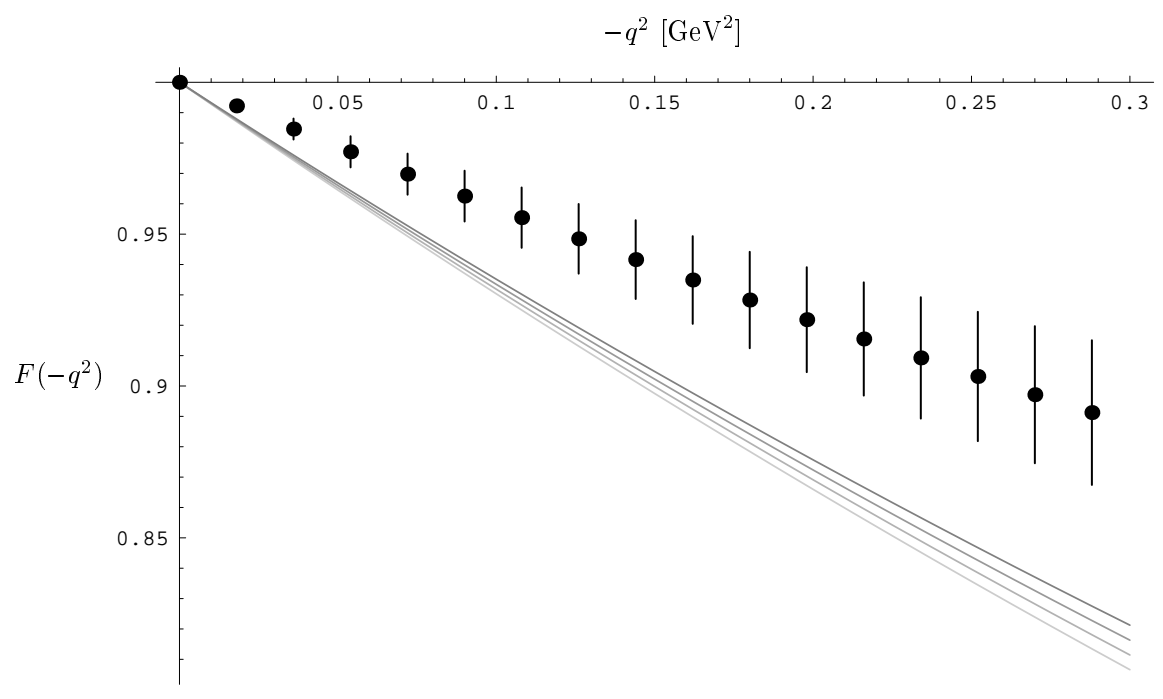


Figure 6
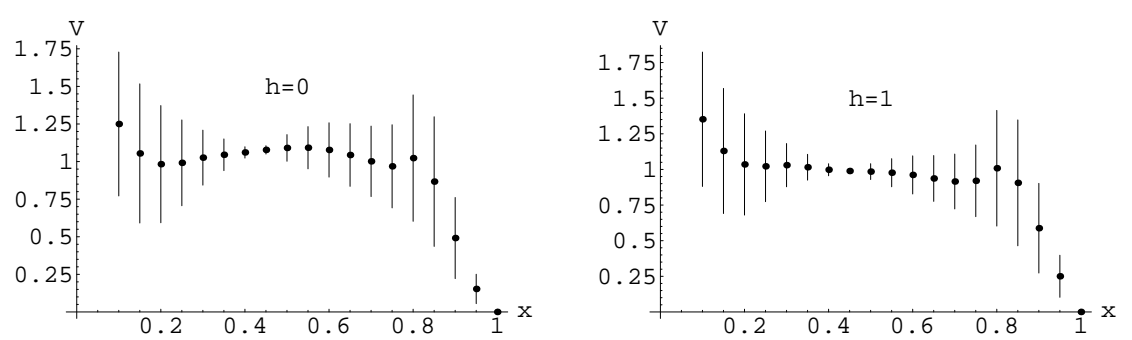
Figure 7

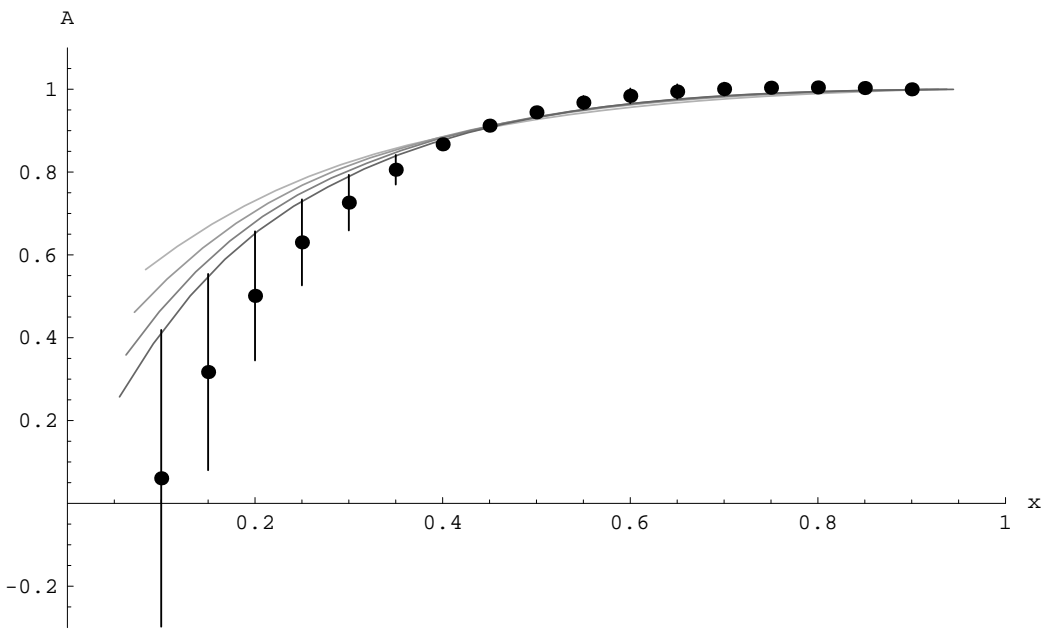

